\title{
Rivaroxaban: striking two birds with one stone
}

\author{
Yae Min Park
}

Division of Cardiology, Department of Internal Medicine, Gachon University Gil Medical Center, Incheon, Korea

Received: June 15, 2021

Accepted: June 20, 2021

\section{Correspondence to}

Yae Min Park, M.D.

Division of Cardiology,

Department of Internal

Medicine, Gachon University

Gil Medical Center, 21

Namdong-daero 774beon-gil,

Namdong-gu, Incheon 21565,

Korea

Tel: $+82-32-460-3663$

Fax: +82-32-469-1906

E-mail:ypruimin@gmail.com https://orcid.org/0000-00033597-9768

\section{See Article on Page 906-913}

Atrial fibrillation $(\mathrm{AF})$ is a common cardiac arrhythmia that is associated with an increased risk of ischemic stroke. $\mathrm{AF}$ accounts for $15 \%$ of strokes overall and $30 \%$ of strokes in patients over 80 years old [1]. Anticoagulation therapy is effective for stroke prevention in patients with non-valvular $\mathrm{AF}$ and is recommended for patients at increased risk. Prior to the use of new oral anticoagulants (NOACs), warfarin was used to prevent embolic stroke in AF patients. However, warfarin treatment requires frequent coagulation monitoring and dose adjustments, making its use in clinical practice difficult [2].

Randomized controlled trials (RCTs) have demonstrated favorable efficacy and safety profiles for NOACs (dabigatran, rivaroxaban, apixaban, edoxaban) compared to warfarin [3-6]. Rivaroxaban is a promising direct factor $\mathrm{Xa}$ inhibitor that may provide more consistent and predictable anticoagulation than warfarin [7]. In the Rivaroxaban Once Daily Oral Direct Factor Xa Inhibition Compared with Vitamin K Antagonism for Prevention of Stroke and Embolism Trial in AF (ROCKET AF), patients were randomly assigned to receive a fixed dose of rivaroxaban (20 or $15 \mathrm{mg}$ daily in patients with a creatinine clearance of 30 to $49 \mathrm{~mL} / \mathrm{min}$ ). Rivaroxaban was non-inferior to warfarin for the prevention of stroke or systemic embolism (SE). Rates of major bleeding were similar for both treatments. Rivaroxaban treatment was associated with reduced intracranial and fatal bleeding and increased transfusions and hemoglobin levels $\geq 2 \mathrm{~g} / \mathrm{dL}$ or more. Major gastrointestinal bleeding was more common in the rivaroxaban group [3]. In the ROCKET AF East Asian cohort, rivaroxaban was associated with reductions in critical organ bleeding and intracranial hemorrhage relative to warfarin. A significant interaction between treatment and residence within or outside East Asia was detected for intracranial hemorrhage. The increased rate of intracranial hemorrhage among East Asian patients receiving warfarin is likely to underly this result. Rivaroxaban was also associated with fewer transfusion events than warfarin in the East Asian cohort [8]. Although rivaroxaban safety issues were of concern in ROCKET AF, a sub-analysis suggested a favorable safety profile in East Asian patients.

In routine clinical practice, NOACs are used in ways other than described in their respective pivotal, phase III RCTs. These cases offer valuable insight into the effectiveness and safety of NOACs used outside of a well-controlled clinical trial. Xarelto for Prevention of Stroke in Patients with AF in Asia (XANAP) is the first prospective, observational study to investigate the safety of rivaroxaban use in rou- 
tine clinical practice in Asia, including Korea [9]. The objective of the XANAP Korea was to investigate the safety and effectiveness of rivaroxaban use in patients with non-valvular AF. In this issue of the Korean Journal of Internal Medicine, Shim et al. [10] showed a consistent safety profile of rivaroxaban in non-valvular AF patients for the prevention of stroke and non-central nervous system SE (non-CNS SE). The findings support the use of rivaroxaban to prevent stroke/non-CNS SE in Korean AF patients

A total of 844 patients were enrolled in the Korean portion of the XANAP study. The mean $\mathrm{CHA}_{2} \mathrm{DS}_{2}$-VASc score was 3.8 and $47 \%$ of the patients had experienced prior stroke, non-CNS SE or transient ischemic attack. Adjudicated treatment-emergent major bleeding developed in $0.8 \%$ of patients (1.1 per 100 patient-years). Death was observed in $1.2 \%$ of the patients. The incidence of non-major bleeding and thromboembolic events were $8.4 \%$ (11.6 per 100 patient-years) and 1.5\% (2.0 per 100 patient-years), respectively. Interestingly, a higher incidence of treatment-emergent bleeding was observed in patients on $15 \mathrm{mg}$ rivaroxaban compared with $20 \mathrm{mg}(10.5 \%$ vs. $8.3 \%)$. This is may be attributed, in all likelihood, to the greater pre-existing co-morbidities of patients taking $15 \mathrm{mg}$ and a preference by the attending physician for the reduced dose, despite the dosing criteria being based solely on creatinine clearance.

XANAP Korea included high-risk patients with a prior history of stroke/non-CNS SE/transient ischemic attack and a high $\mathrm{CHA}_{2} \mathrm{DS}_{2}$-VASc score compared to XANAP and Xarelto for Prevention of Stroke in Patients with AF (XANTUS) [11]. XANAP Korea revealed a high rivaroxaban safety profile and efficacy even in high-risk patients. The incidence of thromboembolic events was comparable to previous XANTUS and ROCKET AF studies and somewhat lower than XANAP, although direct comparison was not possible. XANAP Korea showed an excellent safety profile and $99 \%$ of patients receiving rivaroxaban did not experience any treatment-emergent major bleeding. There were no new adverse events or serious adverse events observed.

An important concern of this study is the relatively high proportion of patients (48.7\%) with missing renal function records. Thus, it is unknown if those patients were dosed as per label recommendation as started in the journal. Since the dosing decision was at the sole discretion of the attending physician, these results may be more indicative of a "real world" clinical practice.

Recent guidelines strongly recommend oral anticoagulant therapy based on validated stroke risk scores, and NOACs are recommended over warfarin in eligible non-valvular AF patients [12]. The Asian population has been reported to have a higher risk for stroke and a greater stroke-related morbidity and mortality $[8,13]$. Bleeding events and rates of intracranial hemorrhage are higher in Asians receiving warfarin [14]. Therefore, the use of rivaroxaban in Korean AF patients with high risk is promising in terms of safety for the prevention of stroke and non-CNS SE. Further studies are required to determine the long-term outcomes of rivaroxaban treatment in a broad clinical setting.

\section{Conflict of interest}

No potential conflict of interest relevant to this article was reported.

\section{REFERENCES}

1. Wolf PA, Abbott RD, Kannel WB. Atrial fibrillation: a major contributor to stroke in the elderly. The Framingham Study. Arch Intern Med 1987;147:1561-1564.

2. Piccini JP, Hernandez AF, Zhao X, et al. Quality of care for atrial fibrillation among patients hospitalized for heart failure. J Am Coll Cardiol 2009;54:1280-1289.

3. Patel MR, Mahaffey KW, Garg J, et al. Rivaroxaban versus warfarin in nonvalvular atrial fibrillation. N Engl J Med 2011;365:883-891.

4. Connolly SJ, Ezekowitz MD, Yusuf S, et al. Dabigatran versus warfarin in patients with atrial fibrillation. N Engl J Med 2009;361:1139-1151.

5. Granger CB, Alexander JH, McMurray JJ, et al. Apixaban versus warfarin in patients with atrial fibrillation. N Engl J Med 2011;365:981-992.

6. Giugliano RP, Ruff CT, Braunwald E, et al. Edoxaban versus warfarin in patients with atrial fibrillation. N Engl J Med 2013;369:2093-2104.

7. Kubitza D, Becka M, Wensing G, Voith B, Zuehlsdorf M. Safety, pharmacodynamics, and pharmacokinetics of BAY 59-7939-an oral, direct Factor Xa inhibitor — after multiple dosing in healthy male subjects. Eur J Clin Pharmacol 2005;61:873-880. 
8. Wong KS, Hu DY, Oomman A, et al. Rivaroxaban for stroke prevention in East Asian patients from the ROCKET AF trial. Stroke 2014;45:1739-1747.

9. Kim YH, Shim J, Tsai CT, et al. XANAP: a real-world, prospective, observational study of patients treated with rivaroxaban for stroke prevention in atrial fibrillation in Asia. J Arrhythm 2018;34:418-427.

10. Shim J, On YK, Kwon SU, et al. A prospective, observational study of rivaroxaban for stroke prevention in atrial fibrillation: the XANAP Korea. Korean J Intern Med 2021;36:906-913.

11. Camm AJ, Amarenco P, Haas S, et al. XANTUS: a real-world, prospective, observational study of patients treated with rivaroxaban for stroke prevention in atrial fibrillation. Eur Heart J 2016;37:1145-1153.

12. Hindricks G, Potpara T, Dagres N, et al. 2020 ESC guide- lines for the diagnosis and management of atrial fibrillation developed in collaboration with the European Association for Cardio-Thoracic Surgery (EACTS): the task force for the diagnosis and management of atrial fibrillation of the European Society of Cardiology (ESC) developed with the special contribution of the European Heart Rhythm Association (EHRA) of the ESC. Eur Heart J 2021;42:373-498.

13. Li YG, Lee SR, Choi EK, Lip GY. Stroke prevention in atrial fibrillation: focus on Asian patients. Korean Circ J 2018;48:665-684.

14. Shen AY, Yao JF, Brar SS, Jorgensen MB, Chen W. Racial/ ethnic differences in the risk of intracranial hemorrhage among patients with atrial fibrillation. J Am Coll Cardiol 2007;50:309-315. 